\section{Statin therapy is beneficial for almost all patients with diabetes}

A previously reported meta-analysis conducted by the Cholesterol Treatment Trialists' Collaboration revealed that statin therapy can reduce the incidence of major vascular events-defined as myocardial infarction or coronary death, stroke, or coronary revascularization-in high-risk patients. The same group has now conducted a second meta-analysis and have shown that statin therapy is similarly beneficial to individuals with type 1 or type 2 diabetes mellitus.

Data were analyzed from 18,686 patients with diabetes (1,466 with type 1 diabetes and 17,220 with type 2 diabetes) and 71,370 individuals without known diabetes, who had collectively participated in 14 randomized statin trials. Among the participants who received statin treatment, the 5-year incidence of major vascular events was reduced by about a fifth per $\mathrm{mmol} / \mathrm{l}$ of LDL cholesterol reduction in both patients with diabetes and those without (relative risk $0.79, P<0.0001$, for both). This proportional reduction was significant irrespective of baseline features, which included a history of vascular disease, type of diabetes, and sex. In addition, in patients with diabetes, the reduction was independent of baseline concentrations of LDL cholesterol, HDL cholesterol, and triglycerides. At no point did LDL cholesterol lowering stop being beneficial-there was no low threshold of LDL cholesterol below which patients gained no benefit.

In light of these findings, the authors believe that a statin regimen that achieves a substantial reduction in LDL cholesterol should be considered in all patients with diabetes who are at risk of vascular disease, irrespective of their lipid profile.

Original article Cholesterol Treatment Trialists' (CTT) Collaborators (2008) Efficacy of cholesterol-lowering therapy in 18686 people with diabetes in 14 randomised trials of statins: a meta-analysis. Lancet 371: 117-125

\section{Patients with nephrotic syndrome have a high absolute risk of thromboembolic events}

Data from a number of studies have indicated that patients with nephrotic syndrome (NS) are at an increased risk of thromboembolic events, but the absolute risks of venous thromboembolism (VTE) or arterial thromboembolism (ATE) in this patient population are unknown. To address this lack of information, Mahmoodi et al. assessed the incidences of ATE and VTE in a cohort of 298 patients with NS.

The mean age of the study population was 42 years and $59 \%$ of the cohort were male. During a mean follow-up of 10 years, 29 patients had at least one episode of VTE and 43 patients had at least one episode of ATE. The resulting annual incidence rates for VTE and ATE were $1.02 \%$ and $1.48 \%$, respectively. However, the incidences of VTE and ATE within 6 months of a diagnosis of NS were considerably higher than these data might indicate - the annual incidences of VTE and ATE on the basis of data obtained in the 6 months following NS diagnosis were $9.85 \%$ and $5.52 \%$, respectively. The ratio of proteinuria to serum albumin (a measure of the severity of NS) was significantly associated with the incidence of VTE (hazard ratio 5.6, 95\% Cl 1.2-26.2; $P=0.03$ ), but neither either parameter alone nor their ratio was predictive of ATE. The incidence of ATE was significantly associated with age, sex, hypertension, diabetes mellitus, smoking and estimated glomerular filtration rate $(P \leq 0.02$ for all).

The authors conclude that patients with NS have high absolute risks of VTE and ATE, particularly in the first 6 months after diagnosis.

\footnotetext{
Original article Mahmoodi BK et al. (2008) High absolute risks and predictors of venous and arterial thromboembolic events in patients with nephrotic syndrome: results from a large retrospective cohort study. Circulation 117: 224-230
}

\section{Short-term withdrawal of warfarin carries low risk of thromboembolism}

For patients who require long-term warfarin treatment, interruption of anticoagulation therapy before surgery carries a risk of thromboembolism; however, the precise risk is unknown. Garcia et al. conducted a prospective observational study to quantify the risk of thromboembolism and bleeding in patients who had short-term interruptions in their warfarin therapy regimen while they underwent minor surgery.

The study included 1,024 patients, who experienced a total of 1,293 interruptions in warfarin therapy. The most common reasons 